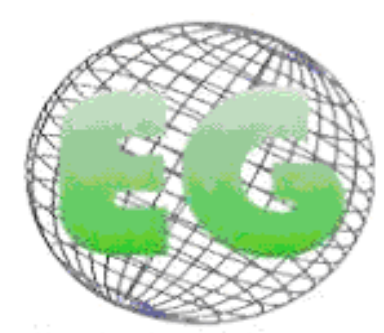

ISSN 1695-6141

$\mathrm{N}^{\circ} 24$

\title{
Valoración del grado de dependencia de los pacientes mayores de 75 años que ingresan en la unidad de traumatología
}

Assessing the degree of dependence of patients aged over 75 admitted to the trauma unit

\author{
*García García, MA., *Tajadura Albillos, MC., "*Gil Millán, P., "Millán Cuesta, B. \\ *Enfermera. Unidad de Traumatología y Ortopedia. Hospital General Yagüe. Burgos. **Enfermera. Hospital \\ Vall d'Hebron. Barcelona.
}

Palabras clave: Dependencia funcional; artrosis; fracturas; Índice de Barthel; envejecimiento. Keywords: Functional dependence; osteoarthritis; fractures; Barthel Index; ageing.

\section{RESUMEN}

El envejecimiento de la población, constituye una de las transformaciones sociales más importantes del último tercio del pasado siglo. Una realidad asociada al envejecimiento es que el número de personas con dependencia aumenta con la edad, derivándose de ello el aumento de la demanda de cuidados por procesos crónicos y degenerativos que lleva asociados.

Se realizó un estudio descriptivo transversal, con el objetivo de medir el grado de dependencia que presenta al ingreso el paciente mayor de 75 años, en la Unidad de Traumatología del Hospital General Yagüe de Burgos y el grado de dependencia con el que abandona el hospital al alta; el período de recogida de datos se efectuó entre enero y junio de 2009.

Para valorar la dependencia funcional utilizamos el Índice de Barthel, además recogimos las variables epidemiológicas, los antecedentes médicos y los datos del ingreso, utilizamos la historia clínica y los datos obtenidos de nuestra labor asistencial. Se realizó análisis de los datos, empleando el paquete estadístico SPSS, encontrando que la edad y el grado de dependencia al alta son estadísticamente significativos, apreciando el mismo comportamiento con otras variables. En el caso de las fracturas constatamos una evolución positiva del Índice de Barthel, negativa en artrosis y sin modificaciones en otras patologías (luxaciones, infecciones...).

Los profesionales de enfermería debemos proporcionar cuidados de calidad, mediante nuestra labor asistencial y docente para mejorar los hábitos de vida del paciente y ayudar a su autonomía. Creemos que el estudio es útil para aplicar cuidados al ingreso y la continuidad de los mismos al alta en coordinación con otros niveles asistenciales.

\section{ABSTRACT}

Ageing of the population has been one of the most important social transformations in the last third of the twentieth century. A reality associated with ageing is that the number of dependent people increases with age. As a result, there is increased demand for care in chronic and degenerative processes. 
We conducted a cross sectional study which aimed to measure the degree of dependency of patients that were admitted to the orthopaedic surgery/ Trauma Unit, General Yagüe Hospital in Burgos when the patient was older than 75 years. We observed dependence when leaving the hospital with medical discharge. Data collection was from January to June 2009

To value the functional dependence we used Barthel's Index. In addition, we gathered epidemiological variables, the medical precedents and the information about the admission. We used the clinical history and the information obtained from our health care assistance.

We performed an analysis of the data, and used an SPSS statistical package. The findings show that age and degree of dependence when the patient is discharged is significant, with same behaviour being appreciated with other variables. In the case of fractures, the evolution is positive in the Barthel Index, osteoarthritis is negative and there were no changes in other pathologies (dislocations, infections...)

Healthcare professionals should provide quality care through our clinical and teaching work, and thus improve the patient's living habits and encourage their independence. Our study is useful if we apply care from the patient being admitted through to discharge in coordination with other levels of care.

\section{INTRODUCCIÓN}

El envejecimiento de la población es la expresión de un logro humano: vivir más y vivir mejor. Constituye unas de las transformaciones sociales más importantes producidas en el último tercio del pasado siglo en los países desarrollados, este fenómeno se ha producido fundamentalmente por la disminución de la fecundidad y los avances tecnológicos que se traducen en una mayor esperanza de vida. ${ }^{(1-3)}$

Pero es logro que requiere implicación social pues el incremento del número de personas mayores y dentro de estas el de las personas de edad más avanzada, ${ }^{(4)}$ coincide en el tiempo con una crisis de los sistemas de apoyo informal que han dado anteriormente respuestas a esas necesidades, como son el cambio en el modelo de familia y la incorporación de la mujer al mundo laboral ${ }^{(5)}$, creándose un problema socio sanitario y económico importante. $(6,7)$

La discriminación por edad supone una percepción negativa de las personas cuando parecen mayores y que muchas veces son estereotipos sociales que no se corresponden con la realidad, ${ }^{(8)}$ ya que también hay ancianos que conservan la capacidad funcional y la autonomía de decisión durante toda su vida e incluso ayudan a la economía familiar cuidando de nietos o encargándose de su pareja dependiente. Es el edadismo o viejísimo, un fenómeno ampliamente extendido en la sociedad actual. ${ }^{(9)}$

El Consejo de Europa en su recomendación R (98) 9 de 1998, ampliada en Oslo el año 2000, define como dependencia "un estado en que las personas, debido a la falta o pérdida de autonomía física, psicológica o mental necesitan algún tipo de ayuda o asistencia para desarrollar sus actividades diarias".

En el año 2002 se añadió que "la dependencia podría estar originada o verse agravada por la ausencia de integración social, relaciones solidarias, entornos accesibles y recursos económicos adecuados para las personas mayores".

La Organización Mundial de la Salud dice que "la esencia misma de la dependencia radica en no poder vivir de manera autónoma y necesitar de forma prolongada la ayuda de otros para las actividades de la vida cotidiana". $(5,10,12)$

Una realidad asociada al envejecimiento es que el número de personas con dependencia aumenta con la edad, derivándose de ello un aumento en la demanda de cuidados por procesos crónicos y degenerativos que lleva asociados. $(1,6,13)$ 
Existe una clara interrelación entre la salud y las situaciones de dependencia En concreto, se tiene constancia de la eficacia de las intervenciones sanitarias en las edades medianas de la vida para prevenir la aparición o retrasar la dependencia en edades más avanzadas. ${ }^{(4,}$ 7, 14)

En España la asimetría existente actualmente entre los servicios sanitarios y los sociales es precisamente una de las dificultades más significativas para la protección de las personas con dependencia. ${ }^{(7)}$

En el modelo de atención a las personas mayores debería plantearse desarrollar medidas para evaluar las necesidades sociales no cubiertas en la población mayor dependiente, ya que nuestro modelo de cuidados tiene un sólido soporte en el apoyo informal, ${ }^{(13,15,16)}$ pero la dependencia ha pasado de ser un problema individual o familiar para convertirse en un problema social que requiere implicación política, ${ }^{(16)}$ ya que la atención y el cuidado de los ancianos debe contemplarse como una etapa vital y no como una carga social. ${ }^{(17)}$

Al margen del impacto que supone la incapacidad sobre la calidad de vida individual y familiar, es también un parámetro de salud ${ }^{(18,22)}$ y por lo tanto predictor de resultados evolutivos relevantes: según se aumenta el nivel de dependencia, se eleva el índice de mortalidad y se aumenta el consumo de recursos sociales con mayor porcentaje de institucionalización, mayor frecuencia de ingresos hospitalarios y mayor estancia media. ${ }^{(7,19)}$ Creemos que la valoración de la dependencia funcional del paciente cuando ingresa en la unidad es de utilidad tanto para planificar los cuidados que precisa durante el ingreso como para la continuidad de cuidados en coordinación con otros niveles asistenciales como atención primaria, centros socio sanitarios y con los cuidadores informales si los tuviera. ${ }^{2,4,}$ $16,19,20)$

Somos conscientes de la dificultad que esto conlleva, sobre todo si no se cuentan con todos los recursos económicos precisos, pero según la Ley de Ordenación de Profesiones Sanitarias (LOPS, 2003) corresponde a los diplomados universitarios en enfermería la dirección, evaluación y prestación de cuidados enfermeros orientados a la recuperación de la salud, así como a la prevención de enfermedades y discapacidades". (21)

\section{OBJETIVOS}

\section{Objetivo principal}

El objetivo principal de este trabajo es comprobar el grado de dependencia que presenta el paciente que ingresa en la unidad de traumatología del Hospital General Yagüe de Burgos y el grado de dependencia con el que abandonan el hospital al alta.

\section{Objetivos secundarios}

Valorar la evolución de la dependencia que presenta el paciente desde el ingreso hasta el alta.

Determinar en qué actividades de la vida diaria se presentan más limitaciones en la autonomía del paciente.

Detectar si los cambios en el grado de dependencia producidos tras el ingreso en el hospital implican una alteración en la organización familiar y social del paciente en el momento del alta hospitalaria. 
Estimar la necesidad de contar con recursos tanto profesionales como materiales y sociales para proporcionar cuidados de calidad.

\section{MATERIAL Y MÉTODO}

Tras una amplia revisión bibliográfica relacionada con el tema se ha realizado un estudio descriptivo transversal en el ámbito de la unidad de traumatología del hospital General Yagüe de Burgos, (sección 4를 izquierda).

La población objeto de estudio la forman los pacientes traumatológicos mayores de 75 años que ingresan en la unidad, que permanezcan en la misma hasta el alta hospitalaria y que voluntariamente ellos o sus familiares acepten participar. Será criterio de exclusión los exitus y los traslados de unidad ya que no será posible hacer la valoración al alta.

\section{Procedimiento:}

La recogida de datos se efectuó desde Enero hasta Junio de 2009.

Para la recopilación de datos se utilizó la historia clínica, los datos que proporciona el paciente o familiar y los que se derivan de nuestra labor asistencial

Para el procesamiento de los datos utilizamos el programa estadístico SPSS v15.

\section{Instrumentos:}

Como instrumentos de recogida de datos se utilizó un cuestionario de elaboración propia (ANEXO I) donde se recogieron variables epidemiológicas como sexo y edad, otras relacionadas con su salud como son si presentaba o no patología asociada, si precisaba intervención quirúrgica y los días de hospitalización, también si antes del ingreso vivía solo, acompañado o en centro socio-sanitario.

En relación al diagnóstico de ingreso se dividió en tres bloques: fracturas, artrosis y otros diagnósticos donde están incluidas infecciones, luxaciones, procesos o patologías a estudios etc.

Para realizar la valoración de la dependencia funcional utilizamos la escala de Barthel (ANEXO II).

El Índice de Barthel (IB) es una medida de la capacidad funcional para realizar las actividades básicas de la vida diaria (ABVD) con demostrada validez y fiabilidad, ${ }^{(16)}$ es fácil de aplicar y de interpretar y no supone ni riesgo ni molestias para los pacientes. ${ }^{2,22)}$ Se trata de asignar a cada paciente una puntuación en función de su grado de capacidad para realizar diez actividades básicas. ${ }^{(23)}$ Se clasifican en tres dimensiones: de autocuidado (alimentación, baño/ducha, arreglo personal, uso de retrete y vestirse); de movilidad (traslado cama-silla, deambulación y subir y bajar escaleras); y funciones corporales (control intestinal y vesical). ${ }^{(19)}$

Los valores que se asignan a cada actividad dependen del tiempo empleado en su realización y de la necesidad de ayuda para llevarla a cabo ${ }^{(22)}$. Son diez actividades a las que se le pueden asignar $0,5,10$ o 15 puntos. El rango global puede variar entre 0 puntos 
(completamente dependiente) y 100 puntos (completamente independiente). De 0 a 90 puntos si usa silla de ruedas.

La escala de Barthel aporta información tanto a partir de la puntuación global como de cada actividad. Esto ayuda a conocer mejor cuáles son las limitaciones específicas de cada persona. ${ }^{(2,16)}$

El profesional de enfermería de la unidad realiza la valoración mediante la escala de Barthel de forma protocolaria a todos los pacientes a partir de los 75 años. Se efectúa la primera valoración en el momento del ingreso y nuevamente cuando se va de alta hospitalaria.

El nivel de independencia funcional obtenido mediante la escala de Barthel no significa necesariamente que pueda vivir solo, ya que no se valoran otras actividades como las funciones cognitivas indispensables para vivir con autonomía. ${ }^{(16)}$

\section{NORMAS ÉTICAS}

Se contó con la aprobación del comité de ética del Hospital General Yagüe de Burgos.

Para la realización del trabajo se respetó el Código Deontológico de la Enfermería Española y las normas de la declaración de Helsinki.

Los pacientes sobre los que se realiza el estudio o sus familiares, facilitaron los datos de forma voluntaria.

\section{RESULTADOS}

Del total de los pacientes traumatológicos mayores de 75 años que ingresaron en la unidad en el periodo de estudio, 195 pacientes se ajustaban a los criterios de inclusión, desestimándose los exitus y los traslados de unidad por no completarse la escala de Barhel al alta.

De los 195 pacientes estudiados, los principales datos se detallan a continuación:

- EDAD: la media de edad ha sido de 83.7 años con una desviación típica de 5.4, con un intervalo de 75 años mínimo y 99 años máximo.

- SEXO: el $28,7 \%$ (n 56) corresponden al sexo masculino y el $71,3 \%$ corresponden al sexo femenino ( $n$ 139) como se puede observar en la figura 1. 


\section{Distribución por sexo}

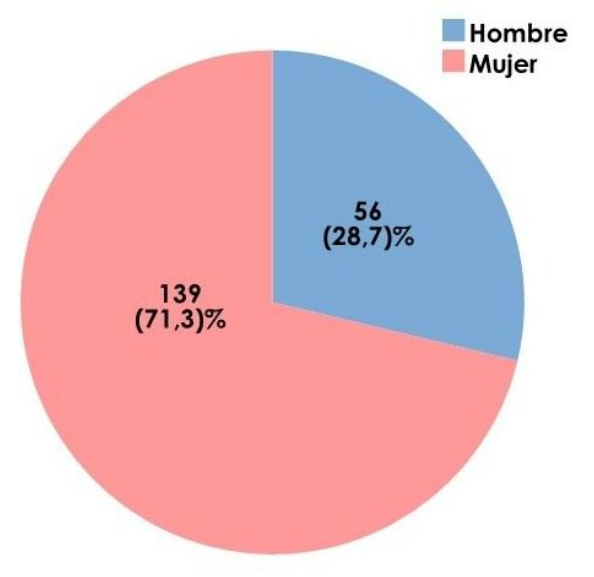

- DIAS DE INGRESO: la estancia media fue de 14 días, con una DE de 7, el intervalo va desde 1 día de mínino hasta 40 días de máximo.

- PATOLOGÍAS ASOCIADAS: se ha encontrado que el 98\% presentaban otras patologías asociadas frente al $2 \%$ que no tenía.

- DIAGNÓSTICO DE INGRESO: en relación al diagnóstico de ingreso correspondió a Fracturas un porcentaje de $67 \%, 16 \%$ a Artrosis y también el $16 \%$ a otros diagnósticos

- TIPO DE INGRESO: el $79 \%$ ingresó de forma urgente y el $21 \%$ de forma programada.

- FRECUENCIA DE IQ: en el $71 \%$ de los casos se procedió a realizar intervención quirúrgica.

- DOMICILIO: con respecto al domicilio previo al ingreso se detectó que un porcentaje del $13 \%$ vivía solo, el 57 \% vivía en familia y el 30 \% vivía en una institución.

\section{DATOS DE LA ESCALA DE BARTHEL}

Se ha cuantificado la puntuación de todas las dimensiones que componen la escala de Barthel y la media al ingreso es de 43 con una desviación típica de 28.5. Al alta, la media es de 50,95 y la desviación típica de 21,4, y como se puede apreciar en la figura 2, la mediana al ingreso de 30 y de 55 al alta y los valores van de 0 de mínimo a 100 de máximo, tanto al ingreso como al alta. 


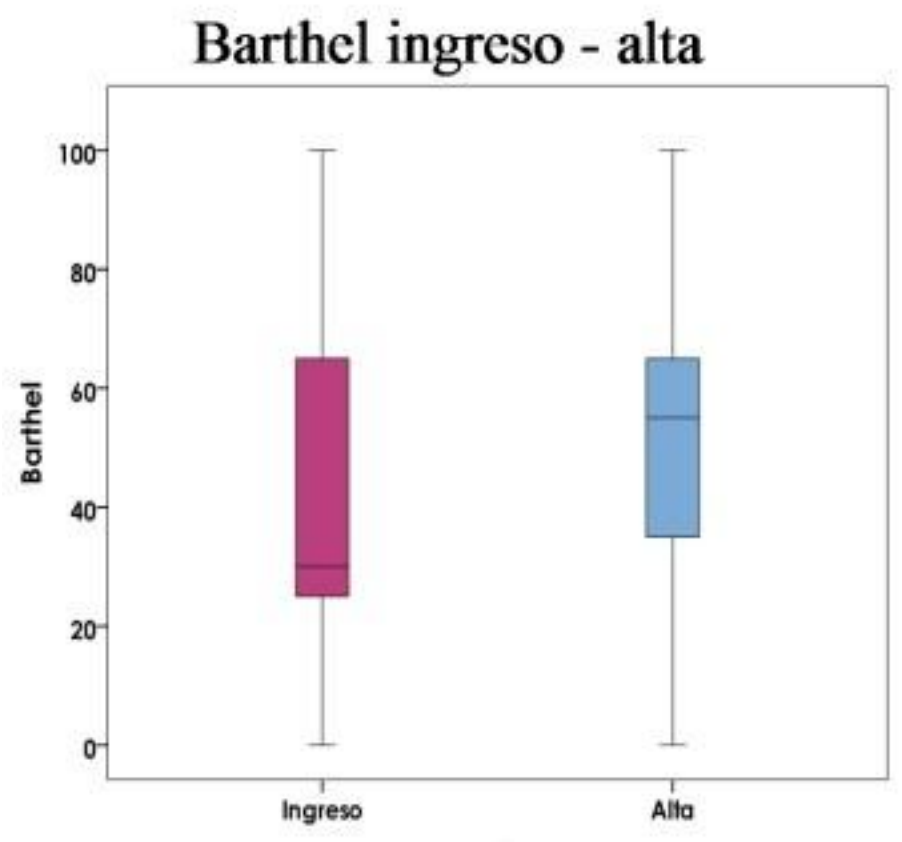

GRADO DE DEPENDENCIA: con respecto al grado de dependencia se obtienen los siguientes porcentajes:

Al ingreso son independientes el 1,5\% (n 3) presentan una dependencia leve el 28\% (n 56), moderada el $14 \%$ (n 28), grave el $33 \%$ (n 64) y dependencia total el $23 \%$ (n44).

En el momento del alta los porcentajes son los que se detallan a continuación:

Son independientes el 0,5\% (n 1), presentan dependencia leve el $35 \%$ (n 69), dependencia moderada también el 35\% ( $n$ 68), dependencia grave el 19\% (n 38) y total el 10\% (n 19).

Estos porcentajes pueden apreciarse en la gráfica $\mathbf{3}$. 


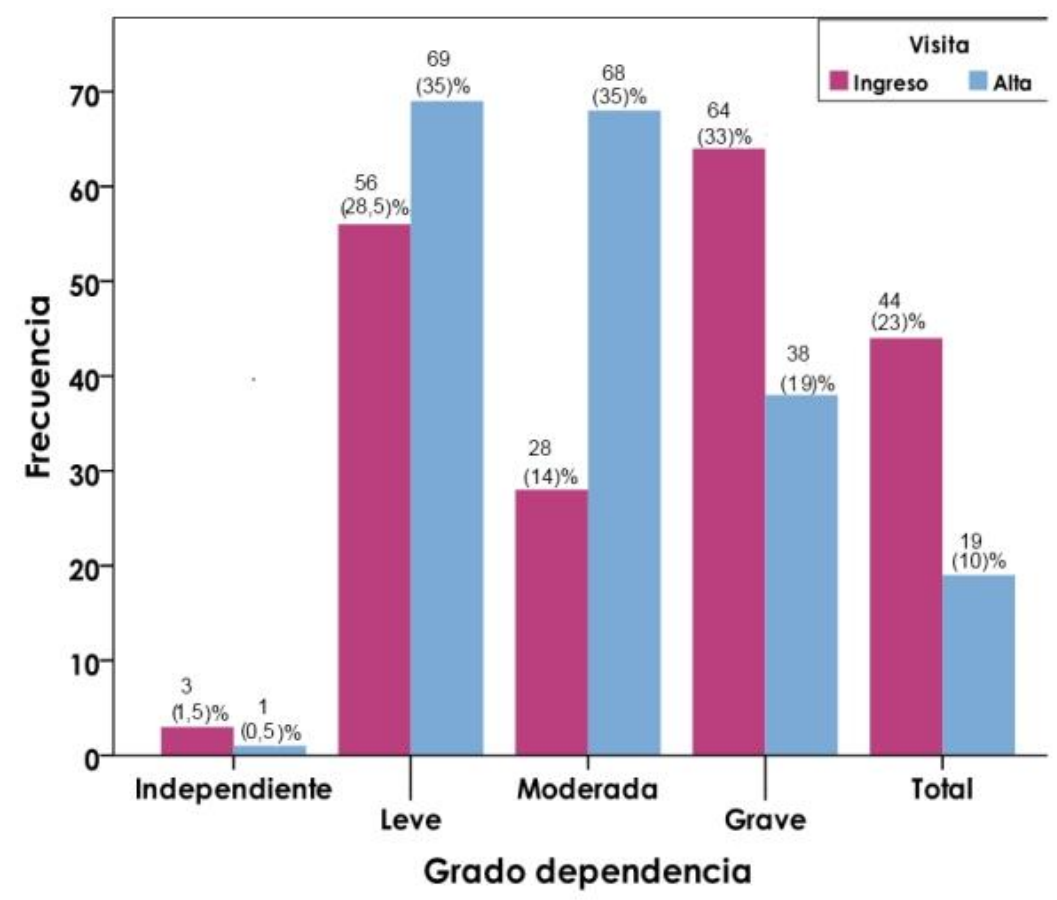

\section{Los porcentajes de las diversas dimensiones de la escala se detallan a continuación:}

- BAÑO: con respecto al apartado del baño el $83 \%$ presentó dependencia y el $17 \%$ presentó independencia al ingreso en esta actividad

Al alta los porcentajes son $87 \%$ y $13 \%$ de dependientes e independientes respectivamente.

- VESTIDO: con respecto al vestido fueron dependientes el $55 \%$, necesitaban ayuda el $30 \%$ y eran independientes el $15 \%$ al ingreso, mientras que al alta los porcentajes se distribuyeron con el $30 \%$ de dependientes, el $67 \%$ que precisaban ayuda y el $4 \%$ que eran independiente

- ARREGLO PERSONAL: los porcentajes que mostraban al ingreso fueron el $80,5 \%$ de dependientes frente al $19 \%$ que tenía necesidad de ayuda y el $0,5 \%$ que era independiente. Los porcentajes que se encontraron al alta fueron de $80,5 \%$ de dependientes y el resto, es decir el 19,5\% necesitaba ayuda. No encontró nadie independiente en esta dimensión.

- TRASLADO SILLÓN-CAMA: se encuentra que en este apartado son dependientes al ingreso el $51 \%$, precisa gran ayuda el $12 \%$, ayuda mínima el $27 \%$ y el $10 \%$ es independiente; en el momento del alta los porcentajes de distribuyen de la siguiente forma: dependientes el $7 \%$, gran ayuda el $42 \%$ mínima ayuda el $45 \%$ e independencia el $6 \%$. (figura 4). 


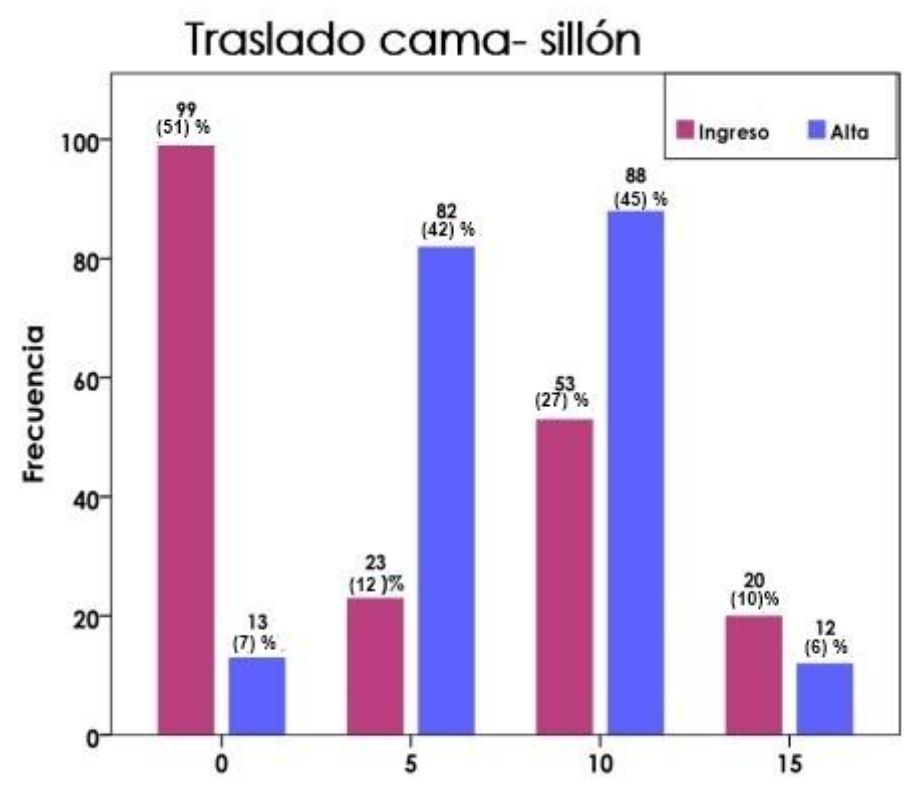

- DEAMBULACION: al ingreso son independientes en este factor el $14 \%$ más el $1 \%$ que es independiente usando silla de ruedas, frente al $56 \%$ de pacientes que son inmóviles, y el $29 \%$ necesita ayuda para caminar.

Al alta los porcentajes son los siguientes: se mantiene el $14 \%$ de independientes mientras que los independientes con silla de ruedas son el $4 \%$, los inmóviles son el 12 . \% y los que precisan ayuda para deambular son el $70 \%$.

Tal y como se refleja en la figura 5 .

Deambulación ingreso - alta

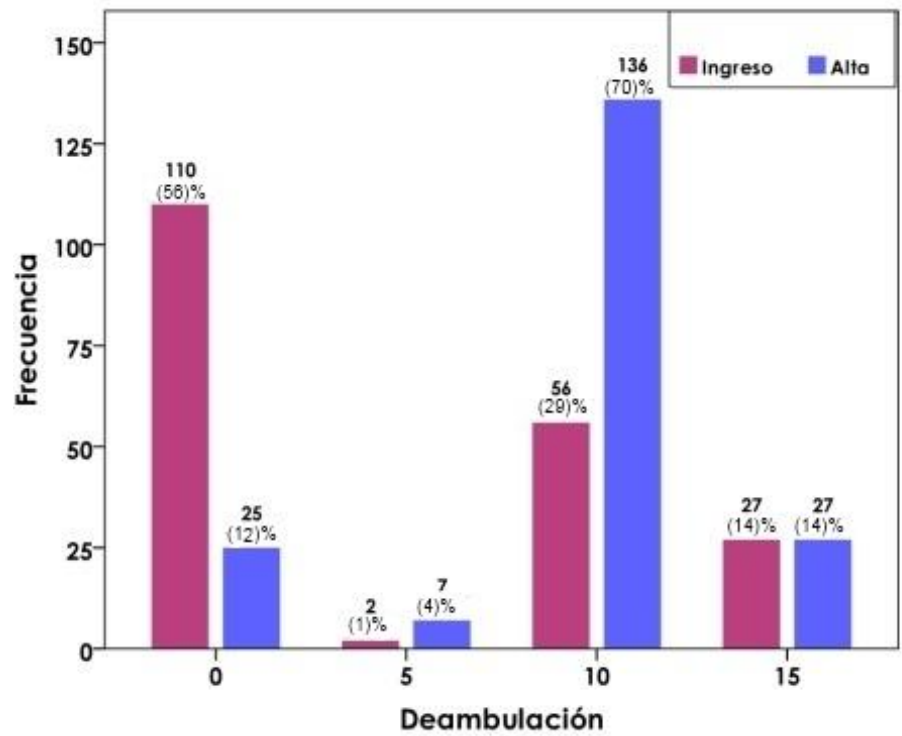


- ESCALONES: con respecto a la posibilidad de subir y bajar escaleras al ingreso los porcentajes son los siguientes: el $63 \%$ era incapaz, el $26 \%$ necesitaba ayuda y el $11 \%$ era independiente. Al alta los porcentajes fueron: incapaz el $61 \%$ necesitado de ayuda el $31 \%$ e independiente el $8 \%$.

- USO RETRETE: al ingreso eran dependientes el 55\%, necesitaban ayuda el $22 \%$ y eran independientes el 23\%. En el momento del alta los porcentajes fueron:30\% dependiente, $53 \%$ precisaban de ayuda y $17 \%$ fueron independientes.

- DEPOSICIÓN: eran incontinentes el $11 \%$, presentaba accidente ocasional el $15 \%$ y era continente el $74 \%$ al ingreso. Al alta fueron incontinentes el $13 \%$, presentaban accidente ocasional el $16 \%$ y fue continente el $71 \%$

- MICCIÓN: con respecto a la micción al ingreso eran continentes el 70\%, incontinentes ocasionales el $15 \%$ y el $15 \%$ también presentaba incontinencia. Al alta los porcentajes fueron: continentes el $67 \%$, incontinentes ocasionales el $16 \%$ e incontinentes el $17 \%$.

- ALIMENTACIÓN: con respecto a la alimentación el 4\% era dependiente, el $61 \%$ precisaba ayuda y el $35 \%$ era autónomo al ingreso, mientras que en el momento del alta el $4 \%$ también era dependiente, el 57\% precisaba ayuda y el 39\% era autónomo.

\section{Relaciones entre las variables}

Para facilitar el procesamiento estadístico se han agrupado los niveles de dependencia en leve y moderada por una parte y grave y total por otra.

Aplicado el estadístico Mc Nemar, se encontró diferencia estadísticamente significativa $(p<0,001)$ entre las variable dependencia ingreso y dependencia alta. (Tabla 1)

\begin{tabular}{|c|c|c|c|c|}
\hline & \multicolumn{2}{|c|}{ Dependencia Ingreso } & \multirow{2}{*}{ P-valor } \\
\hline & & Leve + moderado & Grave + total & \\
\hline \multirow{4}{*}{$\begin{array}{l}\text { Dependencia } \\
\text { Alta }\end{array}$} & lave + madarado & \multirow{4}{*}{$\begin{array}{c}83 \\
42,6 \% \\
4 \\
2,1 \%\end{array}$} & \multirow{3}{*}{$\begin{array}{c}55 \\
28,2 \% \\
53\end{array}$} & \multirow{4}{*}{$<0,001$} \\
\hline & 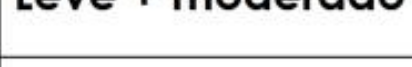 & & & \\
\hline & Grave & & & \\
\hline & Give & & $27,2 \%$ & \\
\hline
\end{tabular}

Realizando un análisis comparativo de cada una de las dimensiones de la escala al ingreso y al alta con el estadístico de Wilcoxon, se encontró significancia estadística $(p<0.00)$ en las dimensiones de traslado cama silla, deambulación, en uso del retrete, micción y baño, también en las actividades de vestido aunque con una significación menor, $(p=0.04)$. En el resto de actividades que valora la escala no hay diferencia estadísticamente significativa entre el valor que presentaba la variable al ingreso y al alta.

\section{Edad y grado de dependencia al alta}

La media de edad en los pacientes con una dependencia leve y moderada fue de 82,7 y la media de edad de los pacientes con un nivel de dependencia grave y total de 86,1 . 
Al relacionar los bloques de edad (< de 85 años por una parte $y>$ de 85 años por otra) y los grados de dependencia al alta (leve y moderada y grave y total) con el estadístico Chi cuadrado el resultado fue estadísticamente significativo con $(p=0,001)$

\section{Sexo y dependencia al alta}

Según los datos obtenidos, el $79 \%$ de los casos de sexo masculino presentaron una dependencia entre leve y moderada y el $21 \%$ entre grave y total, mientras que los casos de sexo femenino los porcentajes fueron del $67 \%$ y $33 \%$ respectivamente. Indicar que hubo un solo caso con independencia y correspondió al sexo femenino.

Relacionado Sexo y Grados de dependencia mediante el estadístico Chi cuadrado no se encontró diferencia estadísticamente significativa, $(p>0,005)$.

\section{Dependencia al alta y domicilio}

Según los datos, de todos los pacientes que vivían solos previo al ingreso, al alta, el $84 \%$ presentó dependencia entre leve y moderada y el $16 \%$ entre grave y total.

De los que vivían acompañados y presentaban dependencia grave o total, el $46 \%$ vivía en institución y el $23 \%$ restante vivía con la familia.

\section{Datos relacionados con el diagnostico de ingreso:}

Encontramos que el porcentaje de dependencia al ingreso con diagnóstico de artrosis es de $6 \%$ de independientes y el $94 \%$ restante presentan dependencia leve.

Los diagnosticados de $\mathrm{Fx}$ al ingreso tienen una dependencia leve el 11\%, moderada el16\%, grave el $41 \%$ y total el $32 \%$.

Con respecto a los otros diagnósticos los porcentajes respectivos son: independiente $3 \%$, dependencia leve $35 \%$, moderada el $23 \%$, grave el $32 \%$ y dependencia total el $7 \%$.

Con respecto a los datos al alta los diagnosticados de artrosis los porcentajes que presentan son $88 \%$ leve, moderada el $9 \%$, grave el $3 \%$.

Los diagnosticados de Fx presentan los siguientes porcentajes:20\%,leve, 41\% moderada, $26 \%$ grave, y total $13 \%$.

Estos datos se pueden apreciar en las graficas 6 y 7 respectivamente. 


\section{Gráfica 6}

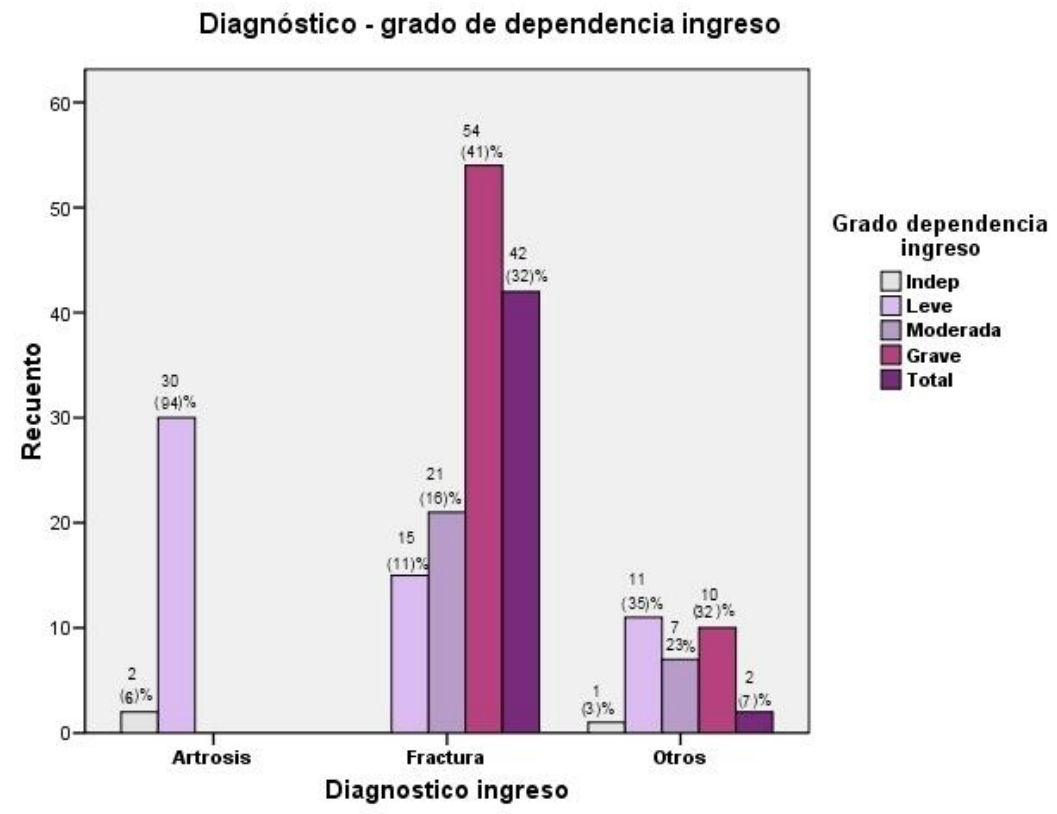

Gráfica 7

Diagnóstico - grado de dependencia alta

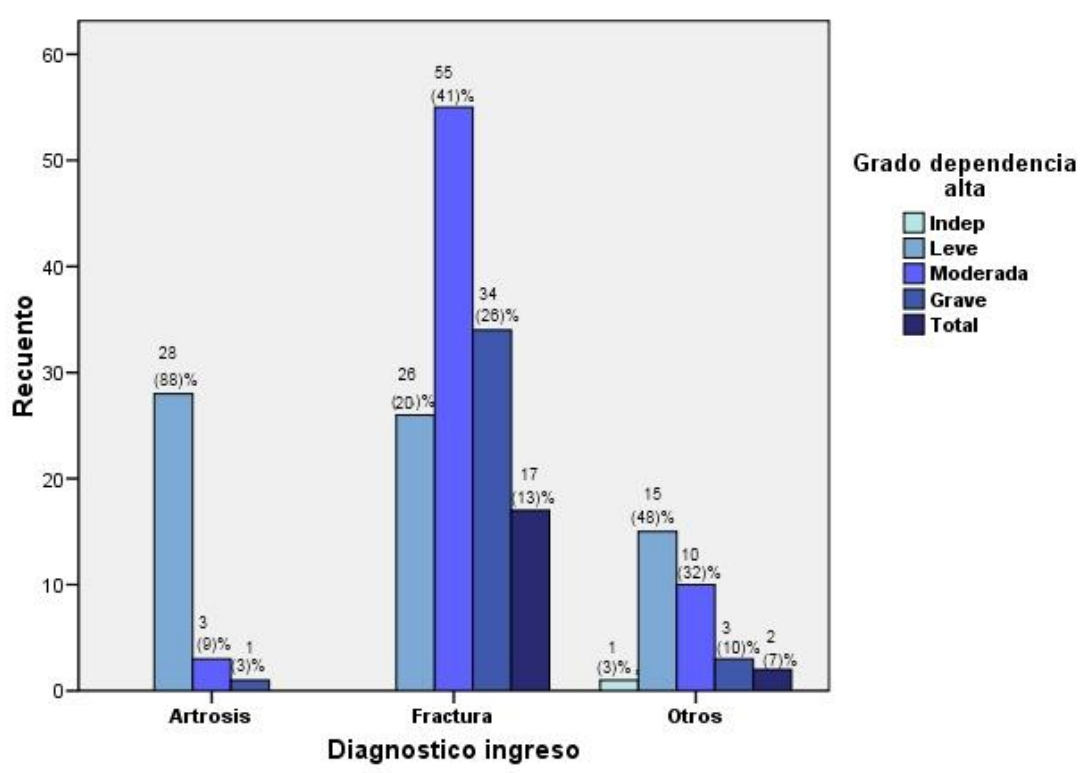

\section{LIMITACIONES}

En el presente trabajo se ha valorado la dependencia funcional, por lo que el valor independiente no significa necesariamente que sea autónomo, ya que puede presentar limitaciones a nivel cognitivo que no ha sido valorado en este trabajo. 
Los datos obtenidos pueden discrepar con el nivel de dependencia de la población general ya que la causa que provocó el ingreso puede favorecer la limitación funcional e incluso no corresponder con los obtenidos con pacientes ingresados con otras patologías no traumatológicas.

El estudio hubiera sido más completo si se hubiera realizado una nueva valoración en las revisiones programadas en las consultas externas o en Atención Primaria.

\section{DISCUSIÓN}

Según los resultados obtenidos en este trabajo destaca la edad elevada de los pacientes estudiados, encontrándose clara relación entre el aumento de la edad y el aumento en el grado de dependencia, coincidiendo con otros estudios realizados en ámbitos diferentes.

No hay diferencia significativa en relación al sexo y nivel de dependencia pero encontramos una mayor representación del sexo femenino en los datos recogidos, así como una media de edad más elevada, sobre todo en el diagnóstico de fracturas, que podría ser consecuencia de una mayor esperanza de vida de las mujeres y tener relación con la osteoporosis producida por alteraciones hormonales, que favorecería la aparición de fracturas en el sexo femenino en edad avanzada.

Según el diagnostico de ingreso nos encontramos una evolución positiva entre el índice de Barthel del ingreso y del alta en el diagnóstico de FX, la evolución es negativa en los ingresos con artrosis y prácticamente no se modifica en otros diagnósticos.

Consideramos lógica esta evolución, ya que la valoración se hace al ingreso y dada la patología traumatológica que presenta la población de este estudio, en el diagnostico de FX, se obtienen puntuaciones muy bajas en los ítems que valoran la movilidad, mejorando notablemente al alta, ocurriendo lo contrario cuando el diagnóstico es de artrosis que por lo general presentan mejor movilidad al ingreso.

Se observa que hay pacientes que vivían solos previo al ingreso y abandonan el hospital con un grado de dependencia que precisará ayuda familiar o soporte social, pudiendo ser esta situación de carácter provisional o permanente. Igualmente se precisará el seguimiento por otros niveles asistenciales para conseguir la mejor y más rápida recuperación.

Creemos que los profesionales de enfermería para proporcionar cuidados de calidad tenemos que ofrecer docencia en nuestra labor asistencial, para mejorar los hábitos de vida del paciente y promocionar su autonomía, ya que el ser humano es un ser social y en todas las etapas de su vida está intercambiando experiencias y conocimientos con su medio, por lo tanto, en la vejez también deben seguir con el proceso de aprendizaje, pero la educación ofrecida tiene que adecuarse a las características especiales de esa etapa de la vida.

También consideramos que es competencia de los profesionales de enfermería formar a los cuidadores informales que así podrán proporcionar mejores cuidados, lo que repercutirá positivamente tanto en el paciente como en el propio cuidador.

Por todo lo anteriormente expuesto, consideramos que se precisan importantes recursos materiales y sociales para proporcionar cuidados de calidad.

Por último, consideramos que se debería seguir realizando estudios sobre la dependencia en otros ámbitos. 


\section{CONCLUSIONES}

La elevada dependencia encontrada en los pacientes estudiados está relacionada con el diagnóstico de ingreso, con las patologías asociadas y con la edad avanzada.

Analizando los resultados de este trabajo se concluye que las actividades más afectadas son aquellas que se relacionan con la movilidad.

Los procesos que provocan el ingreso conllevan un deterioro de la dependencia, aunque sea de forma provisional, por lo que se precisa seguimiento en el momento del alta de otros niveles de atención.

\section{AGRADECIMIENTOS}

A los pacientes que han participado y a todo el personal de enfermería de la unidad de traumatología que ha colaborado ya que sin su ayuda no hubiera sido posible la realización de este trabajo.

A la unidad de docencia, de estadística y de ética e investigación del Hospital General Yagüe por su apoyo en este estudio.

\section{REFERENCIAS BIBLIOGRÁFICAS.}

1 Almeida, Pereira dos Santos, AJ; Costa Pereira Rodrigues VJ. La calidad de vida de la persona de edad avanzada institucionalizada en hogares de ancianos. Rev. Latino-Am. Enfermagem [online]. 2008, vol.16, n.6, pp.1025-1031. Disponible en http:/www.scielo.br/scielo.php?script=sci_arttext\& pid=S010411692008000600014\&Ing=en\&nrm=iso\&tIng=es (Consultado 2009 Nov 20)

2 Alonso Pérez, Laura; Ezkurra Loyoa, Alezne; Amilibia Garaizabal, Libe; González Ignacio, Asunción; Guridi Amenabar, Maite. Nivel de dependencia de los pacientes ingresados. Revista Metas Enferm Fecha de Publicación 2006 oct Año/Volumen/Páginas 9(8):18-24.

3 Triadó, Mํㅡ C (2003) "Envejecer en entornos rurales". Madrid, IMSERSO, Estudios +D+I, no 19. [Fecha de publicación: 13/06/2005].Disponible en http://www.imsersomayores.csic.es/documentos/ documentos/imserso-estudiosidi-19.pdf. (Consultado 2008 Nov 19)

4 Ruiz Miralles ML et al. Estudio de la calidad de vida en las personas mayores confinadas en el domicilio en un barrio de Alicante. Enferm.Clin.2007; 17(6):287-92.Disponible en http://www.doyma.es/revistas/ctl_servlet?_f=7216\&articuloid=13113085 (Consultada 2008 Nov 20)

5 Ministerio de trabajo y asuntos sociales. Atención a las personas en situación de dependencia Libro Blanco .Madrid 23 de Diciembre de2004 disponible en http://www.feafes.com/FEAFES/

DocumentosElectronicos/Capitulo2/Libro+blanco+dependencia.htm (citado 2009 Ene 2)

6 Otero, A ; Zunzunegui, MV ; Rodríguez-Laso, A; Aguilar, MD; Pablo Lázaro, P. Volumen y tendencias de la dependencia asociada al envejecimiento en la población española. Rev Esp Salud Pública 2004 [revista en la Internet]. 2004 Abr; 78(2): 201-213. Disponible en: http://scielo.isciii.es/ scielo.php?script=sci_arttext\&pid=S113557272004000200007\&lng=es. [citado 2010 Jul 29] 
7 Gómez Pavón, J; Martín Lesende, I; Baztán Cortés, JJ; Regato Pajares,P; Formiga Pérez, F; Segura Benedicto ,A; Abizanda Soler, P; De Pedro Cuesta, J .Prevención de la dependencia en las personas mayores. Revista clínica española: publicación oficial de la Sociedad Española de Medicina Interna, ISSN 0014-2565, Vol. 208, №. 7, 2008, pags. 361-362 disponible en http://www.doyma.es/ revistas/ctl_servlet?_f=7216\&articuloid=13124318\&revistaid=65 (Consultado 2009 Ene 2)

8 López Doblas, J; Díaz Conde, MP; (2007). "Aspectos sociológicos del envejecimiento". Madrid, Portal Mayores, Informes Portal Mayores, no 73. Lecciones de Gerontología, XIV [Fecha de publicación: 14/06/2007]. disponible en <http://www.imsersomayores.csic.es/documentos/documentos/lopez-aspectos-01.pdf (consultado 2008 Nov 14)

9 Barcena Calvo, C; Iglesias Guerra JA; Galán Andrés, MI; Abella García,V. Dependencia y edadismo. Implicaciones para el cuidado. Rev enferm.CyL. Vol 1-№ 1 (2009) 46-52. Disponible http://www.revistaenfermeriacyl.com/index.php/revistaenfermeriacyl/article/view/18/10.(Con sultado 2010 Feb 17)

10 Planas Campmany, C. Aportación enfermera a la atención a la dependencia en Cataluña. Nursing 2007 Edicion Española. vol 125 nํ 1:60-65.

11 Consejo de Europa. Recomendación R № (98) 9 del comité de ministros a los Estados miembros relativa a la dependencia. (Adoptada por el comité de Ministros el 18 de Septiembre del año 1998) en la 641aㅡ reunión de Delegados de ministros disponible en http://www.imsersomayores.csic.es/ documentacion/biblioteca/registro.htm?id=51023 (Consultado Nov 2008 23).

12 Organización Mundial de la Salud (OMS) Clasificación Internacional del Funcionamiento, de la Discapacidad y de la Salud (CIF). OMS, 2001 Disponible en http://www.sidar.org/ recur/desdi/pau/cif.php (Consultado 2008 Nov 23).

13 Tomás Aznar. C; Moreno Aznar LA, Germán Bes C., Alcalá Nalváiz T., Andrés Esteban E.. Dependencia y necesidades de cuidados no cubiertas de las personas mayores de una zona de salud de Zaragoza. Rev. Esp. Salud Publica [revista en la Internet]. 2002 Jun [; 76(3): 215-226. Disponible en: http://scielo.isciii.es/scielo.php?script=sci arttext\&pid=S1135$\underline{57272002000300006 \& \text { Ing }=\text { es. }}$ [Citado 2010 Jul 29]

14 Portal de Mayores. Área de envejecimiento. Salud y Psicología. Disponible en http://www.imsersomayores.csic.es/salud/psicologia/autonomia/pyr.html\#aut14 (Consultado 2008 Oct 12)

15 Gómez Raposo, Manuela; Macías Colorado, Mํㅡㄹ Eulalia; Rodríguez Vázquez, Mํa del Carmen. Grado de dependencia de la población mayor de 65 años en la Zona Básica de Salud de Ayamonte. Enfermería Comunitaria (rev. digital) 2008, 4(1). Disponible en <http://www.index-f.com/comunitaria/v4n1/ec6634.php> Consultado 2010 Jul 29).

16 Leno González,D; Fernández Mateo.M; Leno González .LG; Lorenzo Guerrero.MJ. Idice de Barthel. ¿adecuado para planificar necesidades al alta hospitalaria? Enfermería Global (revista electrónica) Octubre 2008 № $14: 1-8$ disponible: www.um.es/eglobal/article/view/35901 (consultado $2010 \mathrm{Jul} 20$ ).

17 Pastor. A. La política: El arte de hacer posible lo necesario. Envejecimiento y dependencia. Revista Ocasa 21 Diciembre 2008.Año III Numero 114:46.

18 Abellán García. A; Castejón Villarejo. P; Imserso. Observatorio de personas mayores. Informe 2008. tomo 1. Colección documentos. Serie documentos estadísticos. Capitulo 2. 
Servicios sociales para personas mayores en España. Enero 2008. Disponible en: www.msps.es/va/novedades/docs/ informe2008_I.pdf . (Consultado 2009 Nov 17).

19 Nasaria González. R; Segovia Diez de Leon. MG. Capacidad funcional de adultos mayores en Hospital de Seguridad Social. Desarrollo cientif Enferm. Vol.18 № 1 EneroFebrero,2010. Disponible en http://www.index-f.com/dce/18/18-5.php .(Consultado 2010 Abr 7)

20 Instituto Europeo de Salud y Bienestar Social. Decálogo para la dependencia. Informe Diciembre 2008. Disponible en http://www.institutoeuropeo.es/esp/decalogos.html. (Consultado 2009 Marz 7)

21 Ley de Ordenación de las profesiones sanitarias. Ley 44/2003 de 21 de noviembre. Articulo 7. Boletín Oficial de Estado, № 280. 22 de noviembre de 2003. Disponible en www.boe.es/boe/ dias/2003/11/22/pdfs/A41442-41458.pdf .(Consultado Oct 2008).

22 Trigas Ferrin. M. Índice de Barthel o discapacidad de Marylnd. Actividades Básicas de la vida diaria (ABVD). Medicina Interna. CHU Juan Canalejo. A Coruña. Disponible en www.meiga.info/escalas/IndiceDeBarthel.pdf. (Consultado 2009 Ene 16).

23 Blanca Gutiérrez. JJ; Caraballo Nuñez. MA; Luque Perez. M;Muñoz Segura. R. El aumento de la independencia de los pacientes en el hospital. Enfermería Global (revista electrónica) Junio $2009 \quad$ №16. Disponible en http://scielo.isciii.es/scielo.php?script=sci_arttext\&pid=S1695-61412009000 2000 04\&lng=es. (consultado 2010 Ene 17). 


\section{ANEXO I}

EDAD:

SEXO: $\quad$ HOMBRE $\square$

MUJER

FECHA DE INGRESO:

FECHA DE ALTA:

DIAS DE INGRESO

DIAGNOSTICO DE INGRESO: ARTROSIS

FRACTURAS

OTROS DIAGNOSTICOS

INTERVENCION QUIRURGICA: SI

NO

PATOLOGIAS ASOCIADAS: SI

NO

DOMICILIO: SOLO

FAMILIA

INSTITUCION 


\section{ANEXO II (Escala de Barthel)}

Puntuaciones Originales de la escala de Barthel

\section{Comer}

$0=$ incapaz

5 = necesita ayuda para cortar, extender mantequilla, usar condimentos etc.

10 = independiente (la comida esta al alcance de la mano)

Trasladarse entre la silla y la cama

0 = incapaz, no se mantiene sentado

5 = necesita ayuda importante (una persona entrenada o dos personas), puede estar sentado

10 = necesita algo de ayuda (una pequeña ayuda física o ayuda verbal)

$15=$ independiente.

\section{Aseo personal}

$0=$ necesita ayuda con el aseo personal

5 = independiente para lavarse la cara, las manos y los dientes, peinarse y afeitarse

\section{Uso del retrete}

0 = dependiente

5 = necesita alguna ayuda, pero puede hacer algo sólo

10 = independiente (entrar y salir, limpiarse y vestirse)

\section{Bañarse o ducharse}

0 = dependiente

5 = independiente para bañarse o ducharse

\section{Desplazarse}

0 = inmóvil

$5=$ independiente en silla de ruedas en $50 \mathrm{~m}$

10 = anda con pequeña ayuda de una persona (física o verbal)

15 = independiente al menos $50 \mathrm{~m}$. con cualquier tipo de muleta, excepto andador

\section{Subir y bajar escaleras}

0 = incapaz

5 = necesita ayuda física o verbal, puede llevar cualquier tipo de muleta 
10 = independiente para subir y bajar

Vestirse y desvestirse

0 = dependiente

5 = necesita ayuda, pero puede hacer la mitad sin ayuda

10 = independiente, incluyendo botones, cremalleras, cordones, etc.

\section{Control de heces:}

$0=$ incontinente (o necesita que le suministren enema)

5 = accidente excepcional (uno/semana)

$10=$ continente

\section{Control de orina}

$0=$ incontinente. 0 sondado incapaz de cambiarse la bolsa

5 = accidente excepcional (máximo uno/24 horas).

10 = continente, durante al menos 7 días

\section{Nivel de Dependencia Según Puntuación Total}

Total de 0 a 100 puntos (de 0 a 90 puntos si usa silla de ruedas)

\begin{tabular}{|l|l|}
\hline$<20$ & DEPENDENCIA TOTAL \\
\hline $20-35$ & DEPENDENCIA GRAVE \\
\hline $40-55$ & DEPENDENCIA MODERADA \\
\hline $60-95$ & LEVE \\
\hline 100 & INDEPENDENCIA FUNCIONAL \\
\hline
\end{tabular}


ISSN 1695-6141

๑ COPYRIGHT Servicio de Publicaciones - Universidad de Murcia 